\title{
Inflammatory Conditions Mimicking Tumours In Calabar: A 30 Year Study (1978-2007).
}

\author{
Omotoso $\mathrm{AJ}^{1}$, Emanghe $\mathrm{UE}^{2}$, Nnoli MA ${ }^{1}$, Ekanem $\mathrm{AI}^{1}$ \\ Departments of ${ }^{1}$ Pathology and ${ }^{2}$ Medical Microbiology \&Parasitology, \\ University of Calabar Teaching Hospital, Calabar.
}

\begin{abstract}
Background: Several lesions in the body present as soft tumor mass which often necessitate an incision or excision biopsy for proper histologic diagnosis. It has been noted that several soft tissue tumours turn out to be primarily infective inflammatory conditions. This study is aimed at reviewing these inflammatory soft tissue masses with a view of understanding their aetiology, histologic morphology and anatomic locations.

Methodology: The records of the Department of Pathology, UCTH, were accessed to retrieve relevant clinical information for reviewing all the histologically confirmed inflammatory tumours seen through the period of 1978 and 2007.

Resut: The study shows that onchocerciasis is the highest soft tissue inflammatory lesion in Calabar, constituting 60\% of the total lesions presenting as tumours over the period of study. Histoplasmosis caused by histoplasms capsulatum is also responsible for the tumour lesions seen and was responsible for approximately $20 \%$ of the cases. Other infective conditions include mycetoma, molluscum contagiosum and tuberculosis. All these lesions present as chronic granulomatous/inflammatory lesions with a palpable mass.

Conclusion: A significant number of microbial agents present a diagnostic challenge in the microbiology laboratory which has been seen to account for the surgically removed soft tissue masses in our hospital.

Keywords: Granulomatous, Necrotizing, Non-Necrotizing, Onchocerciasis, Molluscum Contagiosum.
\end{abstract}

\section{Introduction:}

Chronic granulomatous lesions may resemble deep seated tumours resulting from both infectious and non-infectious agents. An organized inflammatory process made up of tissue macrophages or histiocytes which fuse to form multinucleated giant cells define granulomas.

Difficulties in diagnosis encountered both on clinical and laboratory basis often necessitate surgical excision or biopsy of operable lesions for pathological diagnoses.

Granulomata, irrespective of the putative causative agent occur as a result of host response to the antigen. The antigen may be an infectious pathogen, a foreign substance or an unknown agent which fails to respond to the early inflammatory cells such as neutrophils and eosinophils.

The mass of granulation tissue consists of a collection of modified macrophages or epitheloid cells, surrounding lymphocytes, actively growing fibroblasts and giant cells, all organized into a circumscribed formation.

The granuloma may be necrotizing or non-necrotizing in nature. Although necrotizing granuloma denotes an infectious aetiology, non-necrotizing granuloma may not exclude a non-infectious background.

The range of diseases with granulomatous appearance includes tuberculosis, leprosy, leishmaniasis, cryptococcosis, sarcoidosis, rheumatoid arthritis, and berylliosis among others ${ }^{1}$.

Objective:

To review the surgically removed inflammatory lesions that presented as soft tissue masses and to determine the aetiology, morphology and location of the tumours.

\section{Methods:}

Between January 1978 and December 2007 (a 30 year period), we reviewed all soft tissue tumours sent for histological diagnosis in the Department of Pathology of the University of Calabar. Relevant information such as subjects' biometric data, anatomic body sites where the lesions presented and their histological data were extracted and reviewed. The granuloma was classified by their aetiologies into bacterial, parasitic, fungal and viral types.

\section{Results:}

A total of fifty histologically confirmed chronic inflammatory soft tissue masses were seen at the Pathology Department of the University of Calabar Teaching Hospital during the study period. Thirty of the 
tumours were onchocercal masses accounting for $60 \%$ formed the majority with ten tumours due to Histoplasmosis. Table 1 shows the distribution of all the clinical diagnoses.

The tumours were all of infective origin, namely bacterial, parasitic, fungal and viral aetiology. The two bacterial tumours were each a tuberculous and rhinoscleroma granulomas. Thirty tumours were caused by parasites (onchocerciasis and leishmaniasis) while fifteen were of fungal aetiology, namely histoplasmosis, mycetoma and blastomycosis. There were two viral cases caused by molluscum contagiosum.

All subjects of age groups between 0-5 to 66-70years were affected. The 11-15 year age group had the highest (13) number of tumours closely followed by the 6-10 and 0-5 years age groups, with 7 and 6 lesions, respectively. Table 2 shows the age distribution by the aetiology of the tumours in this study.

The anatomic locations of the different granulomas varied widely. The distribution of onchocercal granulomas, which represented the highest number of tumours, is shown by a pie chart.

The onchocercal nodules predominated the biopsied masses and were widely distributed mainly in chest wall (5), two each in the anterior shoulder region, forehead, knee, anterior abdomen, popliteal fossa and one nodule each presenting in the forearm, iliac crest, face, axilla, inguinal region, scapular, gluteus and intra orbital areas. The sites of presentation of eleven nodules were however not indicated in the study.

The lesions found to be histoplasmosis had two masses presenting over the clavicle and the one tumour each found in the shoulder, ankle, hand, periorbital region and anatomic sites were not indicated for four lesions.

All four lesions histologically confirmed as mycetoma presented in the feet while the single tuberculous nodule was a wrist lesion.

While the rhinoscleroma was biopsied from the maxillary antrum, the leishmanal lesion was from the trunk and the blastomycosis nodule was a skin lesion. Two cases of molluscum contagiosum were removed from the foot and shoulder, respectively.

TABLE 1: DISTRIBUTION OF THE TUMOUR

\begin{tabular}{|c|c|c|}
\hline Clinical Diagnosis & Number & Percentage \\
\hline Onchocerciasis & 30 & 60 \\
\hline Histoplasmosis & 10 & 20 \\
\hline Mycetoma & 4 & 8 \\
\hline Molluscum contagiosum & 2 & 2 \\
\hline Tuberculous granuloma & 1 & 2 \\
\hline Leishmaniasis & 1 & 2 \\
\hline Rhinoscleroma & 1 & 2 \\
\hline Blastomycosis & 1 & $\mathbf{1 0 0}$ \\
\hline Total & $\mathbf{5 0}$ & \\
\hline
\end{tabular}

TABLE 2: AGE DISTRIBUTION OF THE INFECTIOUS AGENTS MIMMICKING SOFT TISSUE TUMOURS

\begin{tabular}{|l|l|l|l|l|l|}
\hline Age & Bacteria & Parasite & Fungi & Virus & Total \\
\hline $0-5$ & $\mathbf{0}$ & $\mathbf{3}$ & $\mathbf{2}$ & $\mathbf{1}$ & $\mathbf{6}$ \\
\hline $6-10$ & $\mathbf{0}$ & $\mathbf{3}$ & $\mathbf{3}$ & $\mathbf{1}$ & $\mathbf{7}$ \\
\hline $11-15$ & $\mathbf{0}$ & $\mathbf{8}$ & $\mathbf{5}$ & $\mathbf{0}$ & $\mathbf{1 3}$ \\
\hline $16-20$ & $\mathbf{0}$ & $\mathbf{2}$ & $\mathbf{0}$ & $\mathbf{0}$ & $\mathbf{2}$ \\
\hline $21-25$ & $\mathbf{1}$ & $\mathbf{1}$ & $\mathbf{0}$ & $\mathbf{0}$ & $\mathbf{2}$ \\
\hline $26-30$ & $\mathbf{0}$ & $\mathbf{5}$ & $\mathbf{1}$ & $\mathbf{0}$ & $\mathbf{1}$ \\
\hline $31-35$ & $\mathbf{0}$ & $\mathbf{2}$ & $\mathbf{0}$ & $\mathbf{0}$ & $\mathbf{6}$ \\
\hline $36-40$ & $\mathbf{0}$ & $\mathbf{1}$ & $\mathbf{0}$ & $\mathbf{0}$ & $\mathbf{2}$ \\
\hline $41-45$ & $\mathbf{1}$ & $\mathbf{0}$ & $\mathbf{0}$ & $\mathbf{0}$ & $\mathbf{2}$ \\
\hline $46-50$ & $\mathbf{0}$ & $\mathbf{1}$ & $\mathbf{2}$ & $\mathbf{0}$ & $\mathbf{1}$ \\
\hline $51-55$ & $\mathbf{0}$ & $\mathbf{0}$ & $\mathbf{1}$ & $\mathbf{0}$ & $\mathbf{2}$ \\
\hline $56-60$ & $\mathbf{0}$ & $\mathbf{3}$ & $\mathbf{0}$ & $\mathbf{0}$ & $\mathbf{4}$ \\
\hline $61-65$ & $\mathbf{0}$ & $\mathbf{1}$ & $\mathbf{1 5}$ & $\mathbf{2}$ & $\mathbf{1}$ \\
\hline $66-70$ & $\mathbf{0}$ & $\mathbf{3 1}$ & & $\mathbf{5 0}$ \\
\hline TOTAL & $\mathbf{2}$ & &
\end{tabular}




\section{FIGURE 1: PIE CHART SHOWING PERCENTAGES DISTRIBUTION OF ANATOMIC LOCATION OF ONCHOCERCIASIS}

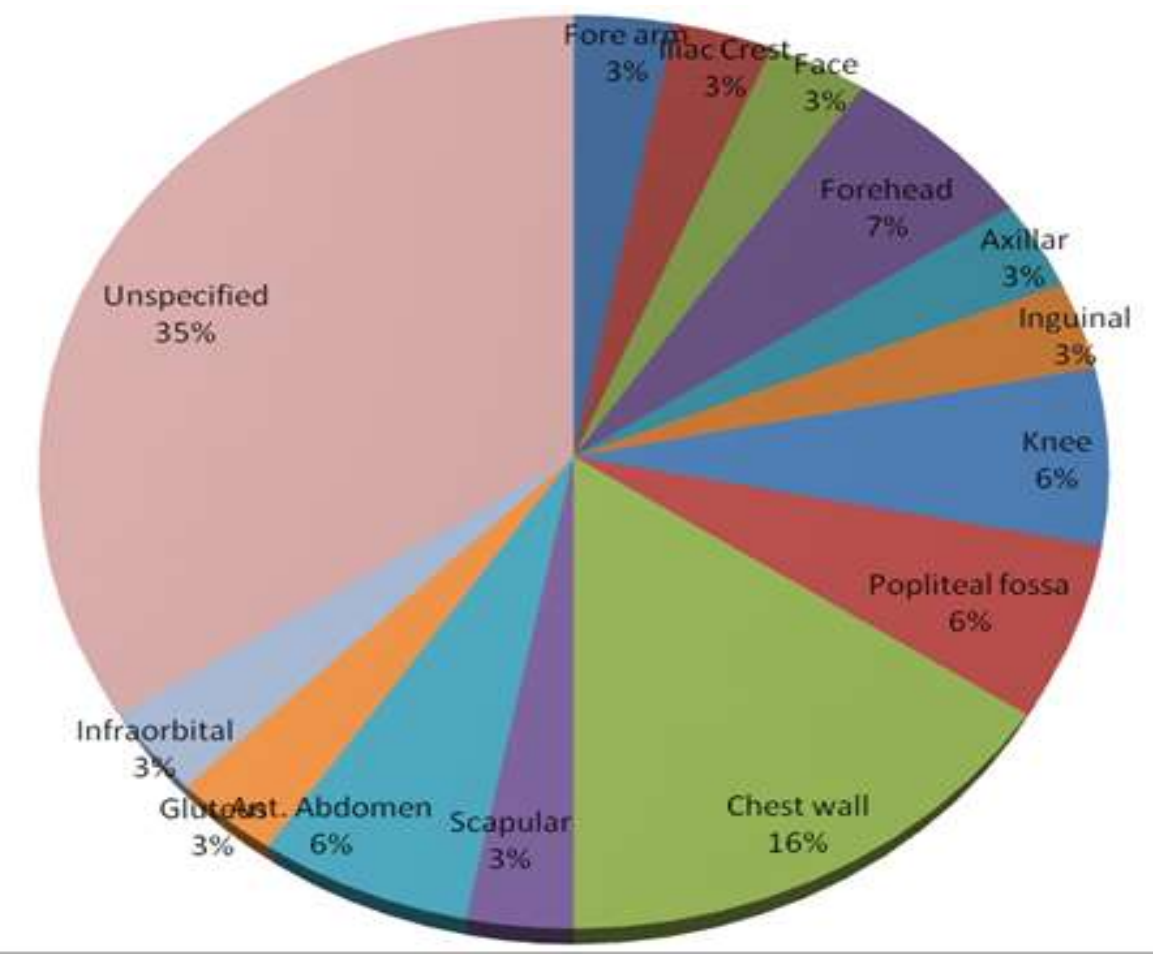

FIGURE 2: PIE CHART FOR ANATOMIC DISTRIBUTION FOR HISTOPLASMOSIS

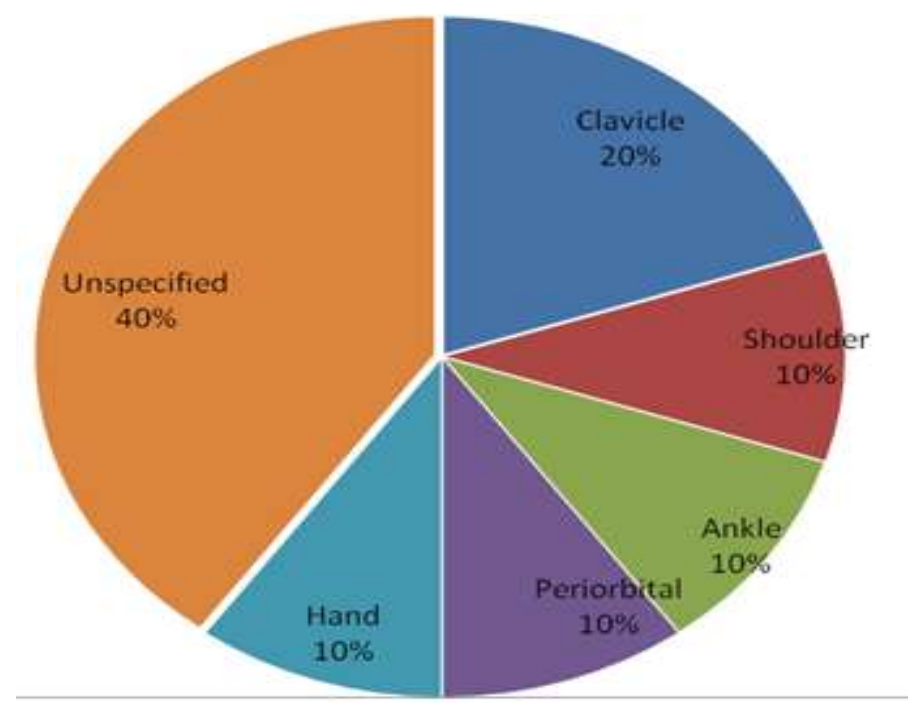

\section{Discussion:}

Inflammatory soft tissue tumours seen in this centre were mainly of infective origin. During the studied period, thirty two thousand, four hundred and forty three $(32,443)$ specimens were received in the Department of Pathology, UCTH and only fifty (50) cases were inflammatory conditions accounting for $0.15 \%$ of all tumours seen within the 30 year study period.

Microbiologic diagnostics difficulties may warrant surgical biopsy for definitive histologic diagnosis. This situation is common in resource-constrained areas with limited capacity for diagnosis of many microbial infections.

Although we had no local studies to compare with respect to sites and age of the tumours, some of them were found in commonly known sites while some were from unusual body sites.

Onchocercal nodules formed around adult worms and wolbachia-carrying microfilaria result in fibrosis ${ }^{7}$. Onchocercal lesions in our study were found below and above the waist with the chest wall dominating. This 
distribution is at variance with the lower torso sites commonly seen in African patients ${ }^{8}$. Although Onchocercal nodules occur more commonly in adults ${ }^{9}, 14$ of the 31 lesions came from children. Similarly, the fungal lesions tended to occur in the young age group of 5-15 years rather than adults.

The two bacterial lesions, tuberculosis and rhinoscleroma were found in adults of age groups 21-25 and 41-45 but it was difficult to assign their distribution. The ages of presentation are typical of the disease.

The two cases of molluscum contagiosum were expectedly from children 10 years and below and occurred at nasal septum, a typical anatomic site ${ }^{4,6}$. Although there is usually a male preponderance of the disease $^{5}$ this study was unable to provide its sex distribution.

The study could not establish in which ages the lone cases of leishmaniasis and blastomycosis fall. For the same reason, the mycetoma cases could not be distributed.

All eight diseases can occur commonly or as sporadic cases in a tropical area such as Nigeria. Also infection with these different aetiologic agents are known to elicit chronic inflammatory reaction leading to the formation of granulomas. ${ }^{1,9,10,11}$

Clinical diagnosis is often a challenge because of the wide range of differential diagnoses for the majority of the tumours in this study. In Nigeria, microbiological diagnoses of fungal and viral diseases is rudimentary while capacity for some bacterial and parasitologic diagnosis of rhinoscleromatis and mycobacterium tuberculosis in non-sputum samples is extremely limited.

\section{Tuberculous Granuloma}

Mycobacterium tuberculosis, a member of the Mycobacterium tuberculosis complex has a long doubling time of between 16 and 20 hours, thus a slow grower. The organism also has intracellular location, which ensures effective evasion of host immune defences.

The host response to eliminate the microbial pathogen results in a fibrotic reaction surrounded by epithelial and chronic inflammatory cells. This lesion or tubercle granuloma consists of a central core of caseating necrotic or non-necrotic tubercles and a peripheral of epithelial cells and histiocytes or macrophages ${ }^{1}$.

The tuberculous granulomata have their nuclei arranged in a semi circle akin of a horse-shoe, called Langhans giant cells. ${ }^{2,3}$

Granulomata are most commonly found in the cutaneous tissue of any part of the body, the pleura and lung tissue.

In this study, one case of tuberculous granuloma presented as a soft tissue tumour in the wrist of the subject, a rather unusual but not unlikely location.

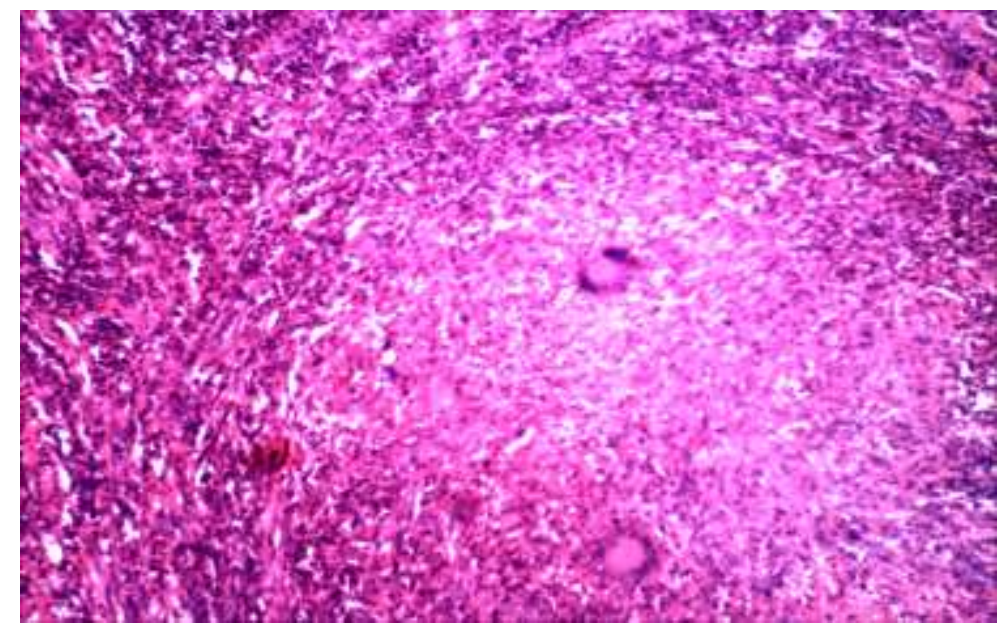

FIGURE 3: H \& E: Section showing central area of caseous necrosis interspersed with multinucleated Langhans' giant cells surrounded by rim of lymphocytes and epitheloid-cells.

Ziehl Neelsen stain, Auramine- Rhodamine stain are some of the common stains for confirmation of the diagnosis of mycobacterium tuberculosis.

\section{Rhinoscleroma}

It is a chronic granulomatous disease almost exclusively limited to the nasal cavity. ${ }^{4}$

The disease is caused by Klebsiella rhinoscleromatis - subspecies of Klebsiella pneumoniae- a gramnegative, encapsulated, nonmotile, rod-shaped bacillus (diplobacillus), member of the Enterobacteriaceae family. It is sometimes referred to as the "Frisch bacillus," named for Anton von Frisch who identified the 
organism in 1882. It is contracted directly by droplets or by contamination of material that is subsequently inhaled.

Scleroma has an insidious and spontaneous onset but progresses through three distinct clinical stages. The nodular or granulomatous stage consists of intranasal polyps. These nodules are painless and may occur at any location between the nostrils and the lung hila.

The disease is seen commonly in young and middle aged adults and persons of lower economic and nutritional levels. It occurs a little more in females than males. ${ }^{5}$

The location within the maxillary antrum in this study is in keeping with the common sites for rhinoscleroma when the nodules spread to the floor, walls and septum of the nose. ${ }^{6}$

\section{Onchocerciasis}

A parasitic infection by the onchocerca volvolus nematode is transmitted by the female blackfly, simulium.

The onchocercal larvae develop in the ensymbiotic wolbachia pipiens worm which resides in the dermis of the human host. The microfilariae develop and mature within nodules formed by the worms.

An inflammatory reaction occurs around the adult worm and that directed against the wolbachia carried by the microfilariae results in tissue fibrosis. ${ }^{7}$

These fibrotic lesions occur as nodules involving skin or subcutaneous tissues. They are usually nontender and of varying sizes located in the pelvis, lower extremities, lower extremities, and lower torso particularly in African patients. ${ }^{9}$

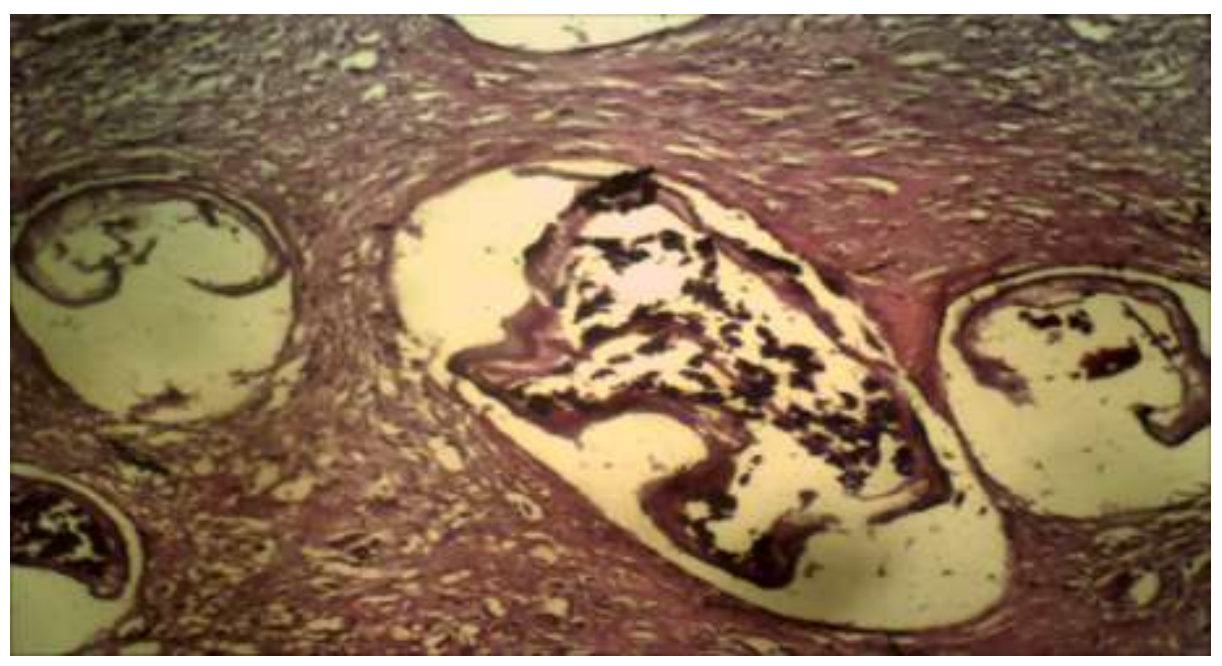

FIGURE 4: Section showing gravid Onchocercal volvulus with fibrosis and hyalinization of the stroma. The stroma is infiltrated by chronic inflammatory cells mainly eosinophils and lymphocytes.

\section{Leishmaniasis}

Cutaneous Leishmaniasis is a chronic parasitic infection caused by different species of leishmania related to the geographic location. In the old world of Asia and Africa, L. major, L. tropica and L. aethiopica are responsible for infection while L. braziliensis, L. mexicana etc are responsible for infection in South America.

Cutaneous leishmaniasis may induce chronic inflammation. ${ }^{10}$

Lesions manifest in skin as dry, crusted and spreading or may be moist and exudative with a red granulating base. A mixture of acute and chronic inflammatory cells of infected and non-infected mononuclear cells predominate with a granulomatous response of epitheloid and giant cells. ${ }^{1}$

\section{Histoplasmosis}

It is a common systemic fungal infection in some parts of North America (mid western and south eastern USA). The disease is caused by Histoplasma capsulatum whose varants and virulence differ with geographic location. ${ }^{14} \mathrm{H}$. capsulatum var dubosii is responsible for most of the disease in Africa with Nigeria, Senegal, Zaire and Uganda accounting for most of the cases.

Infection is acquired by inhalation of fungal spores from the soil, making the lungs the site of primary infection. The lung lesion is a concentric mass of caseating and noncaseating granuloma with a fibrotic thick 
shell. A central calcium deposit within the granuloma differentiates it from a neoplastic mass. Histoplasmosis is increasing in HIV-infected.

Definitive diagnosis is poor by mycological isolation on special media where available in addition to difficulty in obtaining bronchoscopic specimen.

Lesions may be confined or enlarge to erode into airways. Common sites of location are the lung hila or mediastinum, liver or spleen. Skin, bones and joints may be affected.

Histoplasmosis has no known sex or age predilection.

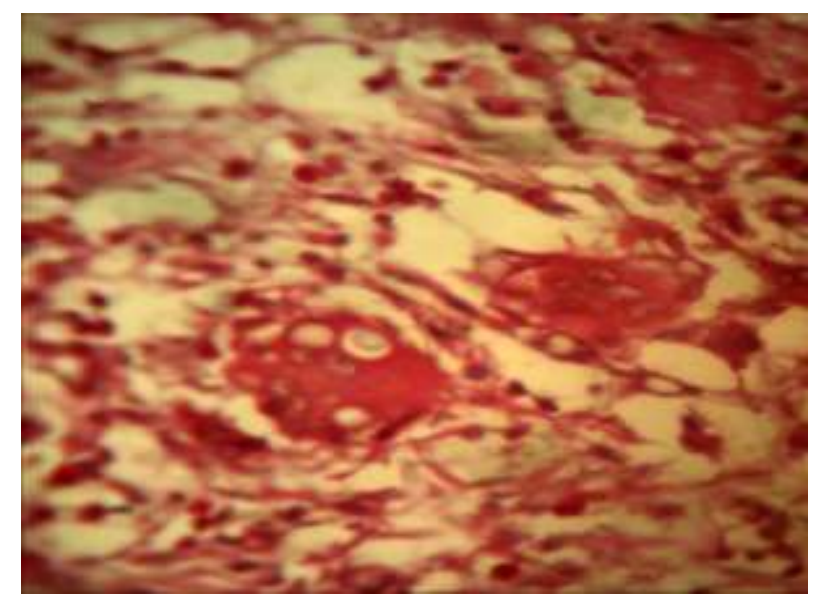

FIGURE 5: Section showing refractile spores of Histoplasma capsulatum within and outside the multinucleated giant cells in the midst of chronic inflammatory cells mainly eosinophils.

\section{Mycetoma}

Mycetoma is a chronic granulomatous disease of the skin and subcutaneous tissue caused by bacteria, actinomycetes or several fungal species.

It is commonly found in the tropic and subtropical regions especially in the Indian subcontinent from where the names Madura foot or Maduromycosis came.

The bacterial and fungal species responsible for this disease vary according to region and the local climate. These include Madurella spp, Pseudallescheria boydii, Acremonium spp, Fusarium spp for the bacterial (Enmycetoma) agents and Actinomydurae madurae, A. pelletieri, Norcardia spp and Streptomyces somaliensis for the Actinomycetoma.

The foot and lower leg are common sites of affectation following traumatic inoculation. However, any part of the body can be affected.

Following inoculation, the organism grows to form masses of fungal mycelia or bacterial filaments. ${ }^{15}$

Mycetoma lesions are granulomatous and are associated with discharging sinuses and deformity of the part of limb. Although it is localized, infection may extend to affect underlying bone.

Adults above 20 years and five times more men than women are commonly affected.

\section{Blastomycosis}

It is a systemic granulomatous lung disease caused by the inhalation of spore forms of the fungus, Blastomyces dermatitidis in moist soil.

The disease is endemic in parts of North America, less commonly in Africa, India, Middle East and Central and South America.

The lung is the primary site of disease from where it may be disseminated to other sites of the body. The skin is the commonest extrapulmonary site, followed by the bone and genitourinary area. ${ }^{16}$

The lung lesion is a noncaseating granuloma of epitheloid and giant cells.

On the skin, lesions are characteristically verrucous, starting as papulopustular and later as heaped-up crusted masses with micro abscesses in the margins.

The skin site of blastomycosis in this study agrees with known location of the extrapulmonary forms of the disease.

\section{Conclusion:}

Microfilariasis caused by onchocerca volvolus and loa loa parasites are common in Cross River State. However, it is onchocerciasis which is often characterized by fugitive swollen lumps in subcutaneous tissues containing dead adult worms. 
The study shows that onchocerciasis is still endemic in the study area with continued transmission of infection from long term carriers. Other lesions seen are histoplamosis, mycetoma, molloscum contagiosum etc.

More study is required to ascertain the pathogenesis of the two infectious agents, histoplasma and onchocerca. Importantly, the finding of high number of onchocerciasis is an indication that community infection is still rife despite efforts of eradication with ivermectin. Also, control efforts may be waning for this parasitic infection as more efforts are concentrated in the emerging public health infections such as HIV.

\section{Reference}

[1] Woodward B H, Rosenberg S I, Farnham R and Adams D O. Incidence and nature of primary granulomatous inflammation in surgically removed material. Am Journal of Surgical Pathology. 1982; 6 (2): 119-129.

[2] Adams, D. O. "The granulomatous inflammatory response. A review". Am J of Pathology. 1976; 84(1): $164-191$.

[3] Granuloma. From Wikipedia, the free encyclopedia. Last modified 6 August, 2011 at 21.52.

[4] Andraea R., Edson RS, Kern EB. Rhinoscleroma: A growing concern in the United States? Mayo Clinic experience. Mayo Clinic Proc. 1993; 68: $1151-1157$.

[5] DiBartolomeo, Joseph R. Scleroma of the nose and pharynx. Western Journal of Medicine. 1976; vol. 124., pp 13-17.

[6] Palmer, PES and Reader MM. The imaging of tropical diseases. 2000. Heidelberg: Springer Verlachi; vols. 1 and 2 (ISBN 3 540 - $66219-7)$.

[7] Saint Andre A., Blackwell NM, Hall LR, et al. The role of endosymbiotic Wolbachia bacteria in the pathogenesis of river blindness. Science. 2002; 295:1892 - 1895. In Mandell, Douglas and Bennett's Principles and Practice of infectious Diseases: $6^{\text {th }} \mathrm{Ed}$, ELSEVIER Churchill Livingstone. Pp. 3273.

[8] American Academy of Paediatrics eBooks; accessed 22 July, 2011 on http: //aaparebook.apphblications.org.

[9] Taylor H R, Duke B O L, Munoz B. The selection of communities for treatment of onchocerciasis with ivermectin. Trop Med Parasitol. 1992; 43:267.

[10] Elder D., Rosalie E., Christine J., Bernett J, Lever's Histopathology of the skin. 1990; 60-61.

[11] John H, Arnold O, Neil P. Textbook of Paediatric dermatology. 2002; vol.1: 516-517.

[12] Rideley D.S. The pathogenesis of cutaneous leishmaniasis. Trans. R Soc Trop. Med Hyg. 1979; 73:150-160

[13] Mandell, Douglas and Bennett's Principles and Practice of Infectious Diseases: $6^{\text {th }}$ Ed, ELSEVIER Churchill Livingstone. Pg

[14] Keath, E.J., Kobayashi G S, Medoff G. Classification of Histoplasma capsulatum by restriction fragrant length polymorphism in a nuclear gene. J Clin Microbiol. 1992; 30:2104-2107.

[15] Manfredi R, Mazzoni A, Nanetti A, et al. Histoplasma capsulatum and duboisii in Europe: The impact of the HIV pandemic , travel, and immigration. Eur J Epidemiol. 1994; 10: 675-681.

[16] Mandell, Douglas and Bennett's Principles and Practice of Infectious Diseases: $6^{\text {th }}$ Ed, ELSEVIER Churchill Livingstone. Pg

[17] Mercurio MG, Eleroski B E. Cutaneous blastomycosis. Cutic 1992; 50:422-424. 